\title{
Wide-field kinematics of globular clusters in the Leo I group ${ }^{\star} \star \star$
}

\author{
G. Bergond ${ }^{1,2,3}$, S. E. Zepf ${ }^{1}$, A. J. Romanowsky ${ }^{4,5}$, R. M. Sharples ${ }^{6}$, and K. L. Rhode ${ }^{7,8}$
}

1 Department of Physics and Astronomy, Michigan State University, East Lansing, MI 48824, USA e-mail: gilles@iaa.es; zepf@pa.msu.edu

2 Instituto de Astrofísica de Andalucía, C/ Camino Bajo de Huétor 50, 18008 Granada, Spain

3 GEPI/CAI, Observatoire de Paris, 77 avenue Denfert-Rochereau, 75014 Paris, France

${ }^{4}$ Departamento de Física, Universidad de Concepción, Casilla 160, Concepción, Chile e-mail: romanow@astro-udec.cl

5 School of Physics and Astronomy, University of Nottingham, University Park, Nottingham, NG7 2RD, UK

${ }^{6}$ Department of Physics, University of Durham, South Road, Durham, DH1 3LE, UK e-mail: r.m.sharples@durham.ac.uk

7 Department of Astronomy, Wesleyan University, Middletown, CT 06459, USA e-mail: kathy@astro.wesleyan.edu

8 Department of Astronomy, Yale University, PO Box 208101, New Haven, CT 06520, USA

Received 24 June 2005 / Accepted 4 November 2005

\section{ABSTRACT}

We present wide-field spectroscopy of globular clusters around the Leo I group galaxies NGC 3379 and NGC 3384 using the FLAMES multi-fibre instrument at the VLT. We obtain accurate radial velocities for 42 globular clusters (GCs) in total, 30 for GCs around the elliptical NGC 3379, eight around the lenticular NGC 3384, and four which may be associated with either galaxy. These data are notable for their large radial range extending from 0.7 to 14.5 ( 2 to $42 \mathrm{kpc}$ ) from the centre of NGC 3379 , and small velocity uncertainties of about $10 \mathrm{~km} \mathrm{~s}{ }^{-1}$. We combine our sample of 30 radial velocities for globular clusters around NGC 3379 with 8 additional GC velocities from the literature, and find a projected velocity dispersion of $\sigma_{\mathrm{p}}=175_{-22}^{+24} \mathrm{~km} \mathrm{~s}^{-1}$ at $R<5^{\prime}$ and $\sigma_{\mathrm{p}}=147_{-39}^{+44}$ at $R>5^{\prime}$. These velocity dispersions are consistent with a dark matter halo around NGC 3379 with a concentration in the range expected from a $\Lambda$ CDM cosmological model and a total mass of $\approx 6 \times 10^{11} M_{\odot}$. Such a model is also consistent with the stellar velocity dispersion at small radii and the rotation of the $\mathrm{H}$ ring at large radii, and has a $(M / L)_{B}$ that increases by a factor of five from several kpc to $100 \mathrm{kpc}$. Our velocity dispersion for the globular cluster system of NGC 3379 is somewhat higher than that found for the planetary nebulae $(\mathrm{PNe})$ in the inner region covered by the PN data, and we discuss possible reasons for this difference. For NGC 3384, we find the GC system has a rotation signature broadly similar to that seen in other kinematic probes of this SB0 galaxy. This suggests that significant rotation may not be unusual in the GC systems of disc galaxies.

Key words. galaxies: elliptical and lenticular, $\mathrm{cD}$ - galaxies: halos - galaxies: kinematics and dynamics - galaxies: star clusters

\section{Introduction}

The current paradigm for galaxy formation is that galaxies form from baryons that cool within dark matter halos. This paradigm is supported by the abundant observational evidence for dark matter in the Universe on many scales, ranging from clusters of galaxies to the halos of individual spiral and dwarf galaxies. Ideally, one would like to test for the presence of dark matter halos around early-type galaxies as well, and to study the properties of these halos.

^ Based on observations collected at the very large telescope (VLT), European Southern Observatory, Chile, under programme 71.B-0537.

$\star \star$ Tables A.1-A.3 are only available in electronic form at the CDS via anonymous ftp to

cdsarc.u-strasbg.fr $(130.79 .128 .5)$ or via

http://cdsweb.u-strasbg.fr/cgi-bin/qcat?]/A+A/448/155
Determining the halo masses and mass profiles of individual elliptical galaxies has proven to be challenging, primarily because they lack a readily observed dynamical tracer at large radii like the H I gas of spiral galaxies. Dedicated spectroscopic studies of the integrated light have been limited to about $R \lesssim 2 R_{\mathrm{e}}$ (e.g., Kronawitter et al. 2000). For the most luminous and massive ellipticals, X-ray observations of the hot gas around these galaxies provide evidence for dark matter (e.g. Loewenstein \& White 1999). Studies of gravitational lensing also indicate the presence of dark matter halos around elliptical galaxies. Specifically, individual cases of strong lensing (e.g., Treu \& Koopmans 2004) show evidence for dark matter at modest radii, while analyses of weak lensing (Wilson et al. 2001; McKay et al. 2001; Hoekstra et al. 2004) provide strong statistical evidence for dark matter halos at large radii around early-type galaxies. Similar results for dark matter at 
large-scales around elliptical galaxies are provided by studies of satellite galaxies in large surveys (Prada et al. 2003; Brainerd 2004). However, for ordinary elliptical galaxies, a substantial range of profiles from inner to outer regions is allowed, and the dependence of halo properties on galaxy luminosity is not strongly constrained. Therefore, determining the masses and mass profiles of ordinary individual elliptical galaxies is of significant interest.

The radial velocities of globular clusters (GCs) and planetary nebulae around elliptical galaxies provide a powerful way to probe the dynamics of their host galaxy. The extended spatial distribution of GC systems makes them particularly useful probes of any dark matter halos around elliptical galaxies. The value of these probes has been demonstrated by the clear evidence for dark matter halos found by studies of the radial velocities of GCs around the elliptical galaxies NGC 4472 (e.g. Zepf et al. 2000; Côté et al. 2003), NGC 4486 (e.g., Cohen 2000; Romanowsky \& Kochanek 2001), NGC 1399 (Richtler et al. 2004), and NGC 5128 (Peng et al. 2004). However, there are only four galaxies with such high quality data, and with the exception of NGC 5128, all are considerably more luminous than a typical elliptical galaxy.

Spectroscopic studies of the planetary nebulae $(\mathrm{PNe})$ can also probe the dynamics of elliptical galaxies. Romanowsky et al. (2003), hereafter R03, specifically carried out a study of the radial velocities of $\mathrm{PNe}$ around three elliptical galaxies of moderate luminosity. They found that the galaxy with the most data, NGC 3379, required little dark matter to the radial limit of their sample at $9 \mathrm{kpc}\left(3^{\prime}\right)$. The other two ellipticals, NGC 821 and NGC 4494 also appeared similar, but with weaker constraints. NGC 3379 (三M105) is in many ways the archetypal ordinary elliptical galaxy, as it is the nearest "normal" giant elliptical galaxy, with a distance of $10 \mathrm{Mpc}$ (Jensen et al. 2003; see also Gregg et al. 2004) in the Leo I group, and an intermediate luminosity of $L_{B}=1.42 \times 10^{10} L_{\odot}\left(M_{B}=-19.9\right)$. Therefore, the possibility that its dark matter halo might not be as expected is critical to follow up.

In this paper, we present the results of a study of the dynamics of the GC system around NGC 3379. Studying the GC system has two important advantages for understanding the halo dynamics of NGC 3379 beyond the obvious utility of providing an independent test of the PNe results. Firstly, there has long been discussion in the literature about the possibility that NGC 3379 has a significantly flattened component viewed face-on (Capaccioli et al. 1991). Subsequent papers have come to mixed conclusions about the presence of such a component (e.g., Statler 1994; Statler \& Smecker-Hane 1999; Statler 2001), but it is obviously an important consideration for interpreting the radial velocities of objects observed in NGC 3379. Here, GCs provide a key advantage, because they are less likely than other tracer populations such as PNe to have significant contribution from a strong disc component. The second advantage of GCs is that candidate GCs can be identified in widefield imaging of nearby ellipticals out to very large radii (e.g. Rhode \& Zepf 2001, 2004 - hereafter RZ04; Harris et al. 2004). This large area over which GCs are observed allows the kinematics of the halo to be probed over a very large radial range.
The plan of the paper is that the spectroscopic observations and the determination of the radial velocities are presented in Sect. 2. The properties of the GC systems of the Leo I group galaxies NGC 3379 and NGC 3384 are discussed in Sect. 3. In Sect. 4, we present the dynamical analysis of the NGC 3379 GC system and the implications for the dark matter halo of this galaxy. The conclusions are given in Sect. 5 .

\section{Observations and data analysis}

\subsection{Identification of globular cluster candidates and MEDUSA fibre allocation}

The advent of large format images with mosaiced arrays of CCDs on 4-m class telescopes allows for the identification of candidate GCs over a wide field around galaxies in the local universe. In particular, RZ04 carried out a multi-colour imaging study of the GC systems of nearby elliptical galaxies utilising the MOSAIC camera on the Mayall $4 \mathrm{~m}$ telescope. They identified GC candidates as unresolved objects with $B V R$ colours consistent with those of Galactic GCs, taking into account the photometric uncertainties (see RZ04 for more details). In order to obtain sufficient $S / N$ spectra in a $\lesssim 4-\mathrm{h}$ exposure, we selected candidates brighter than $V \leq 22$. To help minimise contamination from non-GCs, we also placed a bright limit on the sample of $V \geq 19$, which affects only the extremely high luminosity tail of the GC population at $M_{V} \lesssim-11$. Over the $37^{\prime} \times 37^{\prime}$ MOSAIC field centered on NGC 3379, 196 candidate GCs met these criteria for our spectroscopic follow-up.

We used FLAMES, the multi-object, wide-field fibre spectrograph of VLT/UT2 (see Pasquini et al. 2004) to efficiently obtain spectra of a significant sample of these GCs. The GIRAFFE/MEDUSA mode of this instrument offers 130 fibres over a $25^{\prime}$ diameter field of view, making it an excellent match to the wide-field MOSAIC images (see Fig. 1) and the extended spatial distribution of the globular cluster systems of nearby elliptical galaxies. We ran the FPOSS software to allocate the 130 available fibres to 108 GC candidates, twelve sky positions, and ten $V \sim 18$ stars to be used as templates for radial velocity determinations, in a field centered at $(\alpha, \delta)_{J 2000.0}=\left(10^{\mathrm{h}} 48^{\mathrm{m}} 01^{\mathrm{s}}, 12^{\circ} 33^{\prime} 30^{\prime \prime}\right)$.

\subsection{Observations and radial velocity determination}

The FLAMES observations were carried out in service mode on VLT/UT2 (Kueyen), with the GIRAFFE/MEDUSA configuration. We chose to use the grating LR4 which has a resolving power of $\lambda / \Delta(\lambda) \sim 6000$, and a central wavelength of $\lambda_{\mathrm{c}}=5431 \AA$ that covers many absorption features over $800 \AA$ (including strong $\mathrm{Mg}$ and $\mathrm{Fe}$ lines), and sufficient efficiency to provide useful spectra $(S / N \geq 5$ per pixel) of $V \lesssim 22$ unresolved sources in 3-4 h. Our observing programme consisted of five 2595-s exposures taken on separate nights, in MayJune 2003, under good seeing ( 0.7) conditions.

After standard reductions to extract the spectra with the IRAF HYDRA package, we determined the radial velocities $v$ of the objects by cross-correlating their spectrum with stellar templates. The cross-correlation function $(\mathrm{CCF})$ peak was 


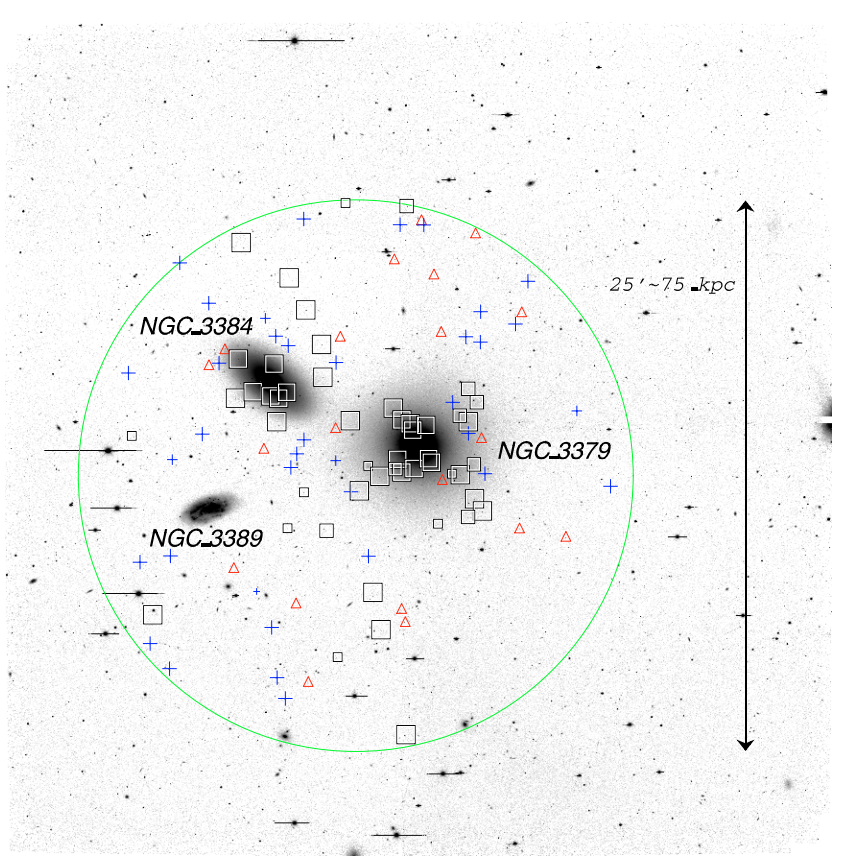

Fig. 1. $V$-band MOSAIC image of the Leo I group. The image covers $37^{\prime} \times 37^{\prime}$ and is plotted with north up and east to the left. The large circle shows the FLAMES $25^{\prime}$ diameter field of view, corresponding to $75 \mathrm{kpc}$ at the distance of NGC 3379. The objects are noted with various symbols according to their FLAMES radial velocities $v$ as follows: squares represent GCs $\left(500<v<1300 \mathrm{~km} \mathrm{~s}^{-1}\right)$ with larger squares indicating a more certain radial velocity, crosses represent objects identified as stars $\left(v<350 \mathrm{~km} \mathrm{~s}^{-1}\right)$ with the size of the symbol corresponding to the confidence in the velocity as above, and triangles show the 21 objects without a clear cross-correlation peak including objects with insufficient $S / N$ and background emission-line galaxies.

fitted by the IRAF/RV task fxcor to estimate the velocity relative to the template, with a typical accuracy of $5-15 \mathrm{~km} \mathrm{~s}^{-1}$ (each pixel represents $0.2 \AA$ or $11 \mathrm{~km} \mathrm{~s}^{-1}$ with the LR4 grating).

The cross-correlation was first done using the ten $V \sim$ 18 red stars to which we allocated fibres so as to provide stellar templates for radial velocity determination. To supplement these "simultaneous" templates, we used 100 F5-K2 high signal-to-noise spectra - both (sub)dwarfs and giants - extracted from the ÉLODIE archive ${ }^{1}$ (Moultaka et al. 2004). The LL_ELODIE stellar library is well-suited for GIRAFFE, with a very similar dispersion (the scale is also of $0.2 \AA /$ pixel, identical to the LR4 grating) which implies minimal rebinning of both spectra.

The CCF peak Gaussian fits to all of the templates were checked both by determining the $\mathcal{R}_{\mathrm{CCF}}$-value of the fit as defined by Tonry \& Davis (1979), and additionally by eye. These agreed well, and the velocities obtained for a dozen of the brightest targets were compared between individual exposure spectra, and also showed good agreement to within the errors estimated by fxcor. We then adopted the following classification of the quality of FLAMES radial velocity determinations:

- class A: more than $98 \%$ of ÉLODIE and more than five (out of ten) simultaneous templates agree within the errors $\delta v$;

\footnotetext{
${ }^{1}$ http://atlas.obs-hp.fr/elodie
}

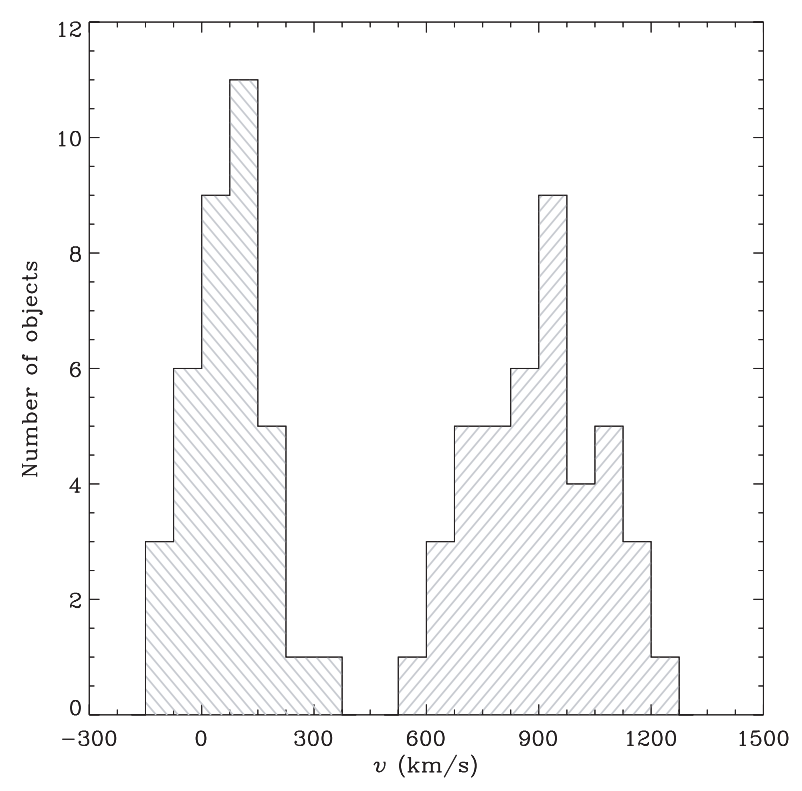

Fig. 2. A histogram of the FLAMES radial velocities for all class A (34 GCs and 32 stars) and class B (8 GCs and 4 stars) objects. This histogram shows a clear $\sim 200 \mathrm{~km} \mathrm{~s}^{-1}$ gap between the highest velocity stars and the lowest velocity clusters (associated with NGC 3384).

- class B: more than $80 \%$ of ÉLODIE and $\geq 3$ simultaneous templates agree within the estimated errors;

- class C (not used): less than 60\% of ÉLODIE and only 0-2 simultaneous templates agree.

For one object, gc771, we clearly detected [O III] $\lambda 5007$ at a radial velocity of $766 \mathrm{~km} \mathrm{~s}^{-1}$. This both provides interesting evidence for a planetary nebula in an extragalactic GC, suggesting these may not be exceedingly rare (see Minniti \& Rejkuba 2002, for another example), and also confirmation of the best estimate for the radial velocity from the cross-correlation of the absorption lines. For all subsequent work, we consider the objects with class A and B radial velocities, and defer consideration of the class $\mathrm{C}$ objects until further data are obtained.

The reliability and accuracy of our radial velocities are strongly supported by a comparison with the data of Puzia et al. (2004, hereafter P04) who used the FORS instrument on the VLT to obtain radial velocities for 18 NGC 3379 inner GCs. Ten of the clusters in the P04 dataset are in our sample, including nine of our class A objects and one class B cluster. The agreement between the two studies is excellent with an average difference of $1 \mathrm{~km} \mathrm{~s}^{-1}$ and a dispersion of $30 \mathrm{~km} \mathrm{~s}^{-1}$. Since $30 \mathrm{~km} \mathrm{~s}^{-1}$ is close to the typical uncertainty in the lower spectral resolution $\mathrm{P} 04$ data, this comparison is consistent with the small uncertainties we find for our velocities of only about $10 \mathrm{~km} \mathrm{~s}^{-1}$ in most cases.

\subsection{Separation of globular clusters and stars}

The histogram of measured radial velocities for the objects we observed is shown in Fig. 2. The heliocentric velocities of NGC 3379 and NGC 3384 are $904 \mathrm{~km} \mathrm{~s}^{-1}$ and $721 \mathrm{~km} \mathrm{~s}^{-1}$ respectively (Falco et al. 1999; Smith et al. 2000) and the galaxies are expected to have modest velocity dispersions. As a 
result, GCs are well separated from stars in the data: there is a $200 \mathrm{~km} \mathrm{~s}^{-1}$ gap between the lowest velocity object we consider a globular cluster and the highest velocity star. Specifically, we classify objects with $500<v<1300 \mathrm{~km} \mathrm{~s}^{-1}$ as GCs, and objects with $-102 \leq v \leq 332 \mathrm{~km} \mathrm{~s}^{-1}$ as stars. The latter range is in good agreement with the expected star field velocity of about $60 \mathrm{~km} \mathrm{~s}^{-1}$ in the direction of NGC 3379 at $(l, b)=\left(233^{\circ} .5,57^{\circ} .6\right)$.

We then have a total sample of 42 GCs with radial velocities, listed in Table 1 along with various properties; 34 of these GCs have highly confident class A radial velocities, and 8 have likely class B determinations. These form the basis for the analysis and discussion in subsequent sections.

We also have radial velocities for 36 stars in our field. This is consistent with expectations from the photometric survey of RZ04, which predicts that about $60 \%$ of our objects at a distance of $4^{\prime}$ from NGC 3379 are GCs, in agreement with the 22 GCs and eleven non-GCs (stars and unclassified objects) within this radius. Most unclassified targets have $V \gtrsim 21$ and are probable galaxies, compact but unresolved in the MOSAIC images (typical seeing 1".3) - including [O II] emission line objects at $0.35 \lesssim z \lesssim 0.55$ for the LR4 wavelength range. The total number of stars and background objects found is consistent with the work of RZ04, given our fibre allocation and $V \leq 22$ limit.

\section{Properties of the globular cluster systems of NGC 3379 and NGC 3384}

\subsection{Identifying globular clusters with NGC 3379 and NGC 3384}

The core of the Leo I group has a compact appearance on the sky with the central elliptical (E1) galaxy NGC 3379 separated by only $7^{\prime}$ from the nearby SB0 galaxy NGC 3384, and by $10^{\prime}$ to the small spiral NGC 3389 , corresponding to projected distances of 20 and $29 \mathrm{kpc}$ respectively. However, the galaxies are fairly clearly delineated in their radial velocities, with NGC 3379 at $904 \mathrm{~km} \mathrm{~s}^{-1}$, NGC 3384 at $721 \mathrm{~km} \mathrm{~s}^{-1}$, and NGC 3389 with $1300 \mathrm{~km} \mathrm{~s}^{-1}$. The velocity for NGC 3379 is the average of the Updated Zwicky Catalogue (UZC, Falco et al. 1999) and Smith et al. (2000) values, and the velocities for NGC 3384 and NGC 3389 are from the UZC. The scatter of measurements in the literature suggests uncertainties of $10-20 \mathrm{~km} \mathrm{~s}^{-1}$ in these numbers.

In Fig. 3 we show the location and velocities of our GC sample overlayed on an image of the Leo I group. This figure shows the clear velocity offset of the group of GCs around NGC 3379, which all scatter around the galaxy's systemic velocity, from the group around NGC 3384, which have velocities like that of NGC 3384 and are thus offset to lower $v$ than NGC 3379 and its GC system. The velocity difference of these two galaxies and their GC systems is useful for determining which clusters in their outer halos belong to which galaxies given their closeness in projection on the sky.

Based on their radial velocities and positions, we assign all of the GCs spatially centered around NGC 3379 to its GC system, along with the GCs located in intermediate spatial regions that have velocities greater than the systemic velocity of NGC 3379, and the globular gc756. We also assign the eight GCs centered on NGC 3384 to this galaxy, and note that these have a strong rotation signature, which we discuss further in Sect. 3.3. We consider the objects gc573, gc571, ad1102, and ad1021 as possibly belonging to either galaxy. We note that it would seem unlikely that NGC 3379 has only outer globulars to its south and not its north, so some of these four would seem likely to belong to NGC 3379 . On the other hand they are slightly closer in projection to NGC 3384, so some of the four would appear likely to be associated with its halo. The velocities of these four GCs are also generally consistent with NGC 3384 (see Fig. 4), but if none of them is assigned to NGC 3379, then its outer halo would have a strange bias towards only globulars with velocities greater than both the systemic velocity of NGC 3379 and its inner GC system (a similar argument supports the identification of gc756 with NGC 3379). We also note that although at very large radii it may become more sensible to consider the halo of the group of galaxies together, given the clear velocity offset of the galaxies and their inner GC systems, a closer relationship to the individual galaxies is indicated over our field of view of $\approx 30 \mathrm{kpc}$.

For the calculations that follow, we use the assignments described above and given in Table 1, and also consider the effect on these calculations of including or excluding various combinations of the GCs with uncertain assignments. As noted below, we find that the conclusions are not significantly affected by the choices in these assignments.

\subsection{Kinematics of the globular cluster system of NGC 3379}

Our sample of NGC 3379 GCs with radial velocities consists of the 30 clusters clearly identified with NGC 3379 and the four for which this assignment is uncertain. To these, we add eight additional GCs from the study of P04 (see Sect. 2.2).

For the sample of 38 GCs associated with NGC 3379, we find a mean of $v=947 \mathrm{~km} \mathrm{~s}^{-1}$ with an uncertainty of $38 \mathrm{~km} \mathrm{~s}^{-1}$ determined via Monte Carlo simulations. The projected velocity dispersion of the full sample is $\sigma_{\mathrm{p}}=169_{-20}^{+20} \mathrm{~km} \mathrm{~s}^{-1}$. We discuss the $\sigma_{\mathrm{p}}$ as a function of radius in the following section, in which we use this to constrain the mass of the halo of NGC 3379. One additional note is that the mean value of the GC radial velocities is $1 \sigma$ larger than the velocity of the galaxy at $904 \mathrm{~km} \mathrm{~s}^{-1}$ (Sect. 3.1). Our GIRAFFE data have good spectral resolution and are highly repeatable from night to night. Moreover, there is excellent agreement between our radial velocities and the independent data of P04. Thus, the most likely explanation is that the small offset is a statistical fluctuation, which can be tested with more data. For modelling purposes, we will use hereafter the mean GC velocity of $937 \mathrm{~km} \mathrm{~s}^{-1}$ from the central regions with good azimuthal coverage (Fig. 4).

Our data also show little evidence for rotation in the NGC 3379 GC system. Although our sample size is modest, even with a much smaller sample we are able to detect rotation in NGC 3384 owing to our small velocity uncertainties (see Sect. 3.3). The exact constraint on the rotation around NGC 3379 depends somewhat on the sample definition, but 
Table 1. FLAMES heliocentic radial velocities $v$ of globular clusters in Leo I: for each of the 42 confirmed GCs, $v$ and its error estimated by fxcor are the median values over the ÉLODIE templates (ordered by projected distance to NGC 3379, $R$ in arcsec; the position angle $\theta$ is counted in degrees from 0 to $90, \mathrm{~N}$ to E). Numbering is from Rhode \& Zepf (2004) as well as the $V$ magnitudes, $B-V$ and $V-R$ colours. Equatorial J2000.0 coordinates should be accurate to $\lesssim 0 \prime^{\prime} 2$ with respect to the GSC2.2. The Tonry \& Davis (1979) cross-correlation coefficient $\mathcal{R}_{\mathrm{CCF}}$ is the median value over the ÉLODIE templates. Class B objects are commented, as well as the 10 GCs in common with Puzia et al. (2004) with their velocity estimate. Full tables of all MEDUSA targets - including stars and galaxies/unclassified objects - are available online.

\begin{tabular}{|c|c|c|c|c|c|c|c|c|c|c|c|}
\hline $\mathrm{Id}_{\mathrm{RZ} 04}$ & $\alpha(\mathrm{J} 2000.0)$ & $\delta(\mathrm{J} 2000.0)$ & $R^{\prime \prime}$ & $\theta^{\circ}$ & $V$ & $B-V$ & $V-R$ & $\mathcal{R}_{\mathrm{CCF}}$ & $v \pm \delta v$ & $\left(\mathrm{~km} \mathrm{~s}^{-1}\right)$ & Comments \\
\hline gc771 & $10^{\mathrm{h}} 47^{\mathrm{m}} 50^{\mathrm{s}} .62$ & $12^{\circ} 35^{\prime} 31^{\prime \prime} .9$ & 39 & 20 & 21.26 & 0.68 & 0.52 & 6.0 & 758 & \pm 9 & Puzia23 at $791 \mathrm{~km} \mathrm{~s}^{-1}$ \\
\hline gc830 & $10^{\mathrm{h}} 47^{\mathrm{m}} 47^{\mathrm{s}} .71$ & $12^{\circ} 34^{\prime} 14^{\prime \prime} .5$ & 49 & 217 & 21.11 & 0.85 & 0.59 & 16.4 & 926 & \pm 5 & \\
\hline gc820 & $10^{\mathrm{h}} 47^{\mathrm{m}} 48.18$ & $12^{\circ} 35^{\prime} 44^{\prime \prime} 9$ & 55 & 336 & 20.94 & 0.75 & 0.49 & 9.9 & 889 & \pm 6 & Puzia27 at $911 \mathrm{~km} \mathrm{~s}^{-1}$ \\
\hline gc764 & $10^{\mathrm{h}} 47^{\mathrm{m}} 51^{\mathrm{s}} .07$ & $12^{\circ} 35^{\prime} 49^{\prime} .1$ & 58 & 21 & 20.00 & 0.72 & 0.52 & 18.7 & 776 & \pm 4 & Puzia24 at $801 \mathrm{~km} \mathrm{~s}^{-1}$ \\
\hline gc839 & $10^{\mathrm{h}} 47^{\mathrm{m}} 47^{\mathrm{s}} .19$ & $12^{\circ} 34^{\prime} 04^{\prime \prime} 6$ & 62 & 217 & 21.98 & 0.69 & 0.46 & 6.7 & 1064 & \pm 13 & \\
\hline gc774 & $10^{\mathrm{h}} 47^{\mathrm{m}} 50^{\mathrm{s}} .40$ & $12^{\circ} 33^{\prime} 47^{\prime \prime} .7$ & 67 & 171 & 21.36 & 0.68 & 0.50 & 5.3 & 656 & \pm 9 & Puzia14 at $645 \mathrm{~km} \mathrm{~s}^{-1}$ \\
\hline gc728 & $10^{\mathrm{h}} 47^{\mathrm{m}} 53^{\mathrm{s}} .42$ & $12^{\circ} 34^{\prime} 12^{\prime \prime} .2$ & 69 & 127 & 21.83 & 0.81 & 0.44 & 5.2 & 978 & \pm 15 & Puzia13 at $941 \mathrm{~km} \mathrm{~s}^{-1}$ \\
\hline gc741 & $10^{\mathrm{h}} 47^{\mathrm{m}} 52^{\mathrm{s}} .60$ & $12^{\circ} 35^{\prime} 59^{\prime \prime} 6$ & 78 & 34 & 21.27 & 0.97 & 0.59 & 9.6 & 1188 & \pm 6 & \\
\hline gc740 & $10^{\mathrm{h}} 47^{\mathrm{m}} 52^{\mathrm{s}} .65$ & $12^{\circ} 33^{\prime} 37^{\prime \prime} 9$ & 88 & 150 & 21.85 & 1.07 & 0.52 & 6.2 & 889 & \pm 7 & Puzia12 at $867 \mathrm{~km} \mathrm{~s}^{-1}$ \\
\hline gc719 & $10^{\mathrm{h}} 47^{\mathrm{m}} 53^{\mathrm{s}} .83$ & $12^{\circ} 33^{\prime} 46^{\prime \prime} 2$ & 92 & 138 & 21.51 & 0.65 & 0.52 & 4.4 & 740 & \pm 10 & Puzia11 at $705 \mathrm{~km} \mathrm{~s}^{-1}$; class B \\
\hline gc709 & $10^{\mathrm{h}} 47^{\mathrm{m}} 54^{\mathrm{s}} .19$ & $12^{\circ} 36^{\prime} 31^{\prime \prime} 5$ & 118 & 35 & 20.97 & 0.72 & 0.50 & 13.0 & 954 & \pm 4 & \\
\hline gc682 & $10^{\mathrm{h}} 47^{\mathrm{m}} 56^{\mathrm{s}} .72$ & $12^{\circ} 33^{\prime} 26^{\prime \prime} 4$ & 137 & 130 & 20.47 & 0.63 & 0.48 & 8.1 & 1137 & \pm 6 & Puzia09 at $1130 \mathrm{~km} \mathrm{~s}^{-1}$ \\
\hline gc923 & $10^{\mathrm{h}} 47^{\mathrm{m}} 41^{\mathrm{s}} .91$ & $12^{\circ} 36^{\prime} 11^{\prime \prime} .2$ & 139 & 303 & 21.97 & 0.75 & 0.57 & 4.0 & 999 & \pm 14 & Class B \\
\hline gc925 & $10^{\mathrm{h}} 47^{\mathrm{m}} 41^{\mathrm{s}} .87$ & $12^{\circ} 33^{\prime} 31^{\prime \prime} 0$ & 144 & 235 & 21.18 & 0.99 & 0.56 & 13.2 & 1048 & \pm 4 & \\
\hline gc948 & $10^{\mathrm{h}} 47^{\mathrm{m}} 40.34$ & $12^{\circ} 35^{\prime} 54^{\prime \prime} 9$ & 152 & 293 & 20.25 & 0.66 & 0.38 & 6.8 & 1264 & \pm 9 & Puzia33 at $1310 \mathrm{~km} \mathrm{~s}^{-1}$ \\
\hline gc958 & $10^{\mathrm{h}} 47^{\mathrm{m}} 39.34$ & $12^{\circ} 33^{\prime} 59^{\prime} 1$ & 164 & 250 & 21.57 & 0.74 & 0.42 & 4.4 & 951 & \pm 8 & Class B \\
\hline gc634 & $10^{\mathrm{h}} 48^{\mathrm{m}} 02^{\mathrm{s}} .13$ & $12^{\circ} 35^{\prime} 57^{\prime \prime} .3$ & 196 & 71 & 20.97 & 0.77 & 0.46 & 7.0 & 950 & \pm 6 & \\
\hline gc971 & $10^{\mathrm{h}} 47^{\mathrm{m}} 38^{\mathrm{s}} .75$ & $12^{\circ} 36^{\prime} 47^{\prime \prime} 8$ & 199 & 305 & 21.78 & 0.83 & 0.47 & 4.4 & 1027 & \pm 13 & Class B \\
\hline gc949 & $10^{\mathrm{h}} 47^{\mathrm{m}} 40.33$ & $12^{\circ} 37^{\prime} 24^{\prime \prime} 1$ & 205 & 317 & 21.81 & 0.69 & 0.43 & 3.0 & 933 & $\pm \quad 27$ & Class B \\
\hline gc645 & $10^{\mathrm{h}} 48^{\mathrm{m}} 00^{\mathrm{s}} .52$ & $12^{\circ} 32^{\prime} 46^{\prime \prime} 9$ & 206 & 128 & 21.02 & 0.78 & 0.48 & 7.5 & 654 & \pm 6 & Puzia05 at $632 \mathrm{~km} \mathrm{~s}^{-1}$ \\
\hline gc960 & $10^{\mathrm{h}} 47^{\mathrm{m}} 39^{\mathrm{s}} .25$ & $12^{\circ} 32^{\prime} 25^{\prime \prime} 6$ & 216 & 226 & 20.06 & 0.81 & 0.49 & 14.9 & 899 & \pm 5 & \\
\hline gc946 & $10^{\mathrm{h}} 47^{\mathrm{m}} 40{ }^{\varsigma} .46$ & $12^{\circ} 31^{\prime} 36^{\prime \prime} 4$ & 241 & 215 & 21.67 & 0.65 & 0.36 & 4.3 & 739 & \pm 6 & Class B \\
\hline gc984 & $10^{\mathrm{h}} 47^{\mathrm{m}} 37^{\mathrm{s}} .80$ & $12^{\circ} 31^{\prime} 52^{\prime \prime} 7$ & 254 & 224 & 20.86 & 0.82 & 0.49 & 7.8 & 1130 & \pm 5 & \\
\hline gc573 & $10^{\mathrm{h}} 48^{\mathrm{m}} 07^{\mathrm{s}} .19$ & $12^{\circ} 37^{\prime} 55^{\prime \prime} 1$ & 318 & 55 & 20.94 & 0.95 & 0.57 & 13.0 & 752 & \pm 4 & NGC 3384 cluster? \\
\hline gc581 & $10^{\mathrm{h}} 48^{\mathrm{m}} 06^{\mathrm{s}} .60$ & $12^{\circ} 30^{\prime} 58^{\prime \prime} 5$ & 346 & 133 & 21.82 & 0.98 & 0.58 & 3.9 & 1064 & \pm 11 & Class B \\
\hline gc571 & $10^{\mathrm{h}} 48^{\mathrm{m}} 07^{\mathrm{s}} .40$ & $12^{\circ} 39^{\prime} 24^{\prime \prime} 1$ & 378 & 45 & 21.25 & 0.73 & 0.50 & 5.9 & 792 & \pm 9 & NGC 3384 cluster? \\
\hline $\operatorname{gc} 482^{a}$ & $10^{\mathrm{h}} 48^{\mathrm{m}} 13^{\mathrm{s}} .88$ & $12^{\circ} 37^{\prime} 14^{\prime \prime} 1$ & 388 & 69 & 21.59 & 1.00 & 0.63 & 17.6 & 908 & \pm 6 & NGC 3384 cluster \\
\hline gc454 & $10^{\mathrm{h}} 48^{\mathrm{m}} 15^{\mathrm{s}} .73$ & $12^{\circ} 35^{\prime} 54^{\prime \prime} .2$ & 394 & 81 & 21.89 & 1.06 & 0.62 & 6.1 & 874 & \pm 8 & NGC 3384 cluster \\
\hline gc461 & $10^{\mathrm{h}} 48^{\mathrm{m}} 15^{\mathrm{s}} .36$ & $12^{\circ} 36^{\prime} 58^{\prime \prime} 0$ & 404 & 72 & 21.87 & 0.71 & 0.45 & 9.4 & 847 & \pm 17 & NGC 3384 cluster \\
\hline gc670 & $10^{\mathrm{h}} 47^{\mathrm{m}} 58^{\mathrm{s}} .08$ & $12^{\circ} 28^{\prime} 11^{\prime \prime} .2$ & 422 & 163 & 20.37 & 0.72 & 0.44 & 7.4 & 921 & \pm 6 & \\
\hline gc442 & $10^{\mathrm{h}} 48^{\mathrm{m}} 16^{\mathrm{s}} .81$ & $12^{\circ} 37^{\prime} 04^{\prime \prime} 0$ & 426 & 72 & 21.15 & 0.74 & 0.51 & 7.7 & 878 & \pm 11 & NGC 3384 cluster \\
\hline gc449 & $10^{\mathrm{h}} 48^{\mathrm{m}} 16^{\mathrm{s}} .20$ & $12^{\circ} 38^{\prime} 30^{\prime \prime} 6$ & 452 & 61 & 20.97 & 0.80 & 0.54 & 15.8 & 672 & \pm 4 & NGC 3384 cluster \\
\hline gc387 & $10^{\mathrm{h}} 48^{\mathrm{m}} 20^{\mathrm{s}} .06$ & $12^{\circ} 37^{\prime} 15^{\prime \prime} .5$ & 476 & 73 & 21.98 & 0.83 & 0.44 & 5.4 & 704 & \pm 11 & NGC 3384 cluster \\
\hline ad1102 & $10^{\mathrm{h}} 48^{\mathrm{m}} 10^{\mathrm{s}} .29$ & $12^{\circ} 40^{\prime} 57^{\prime \prime} .9$ & 476 & 40 & 19.28 & 0.75 & 0.50 & 23.8 & 920 & \pm 3 & NGC 3384 cluster? \\
\hline gc683 & $10^{\mathrm{h}} 47^{\mathrm{m}} 56^{\mathrm{s}} .61$ & $12^{\circ} 26^{\prime} 30^{\prime \prime} 2$ & 514 & 168 & 21.14 & 0.70 & 0.39 & 4.4 & 1087 & \pm 14 & \\
\hline gc351 & $10^{\mathrm{h}} 48^{\mathrm{m}} 23^{\mathrm{s}} .34$ & $12^{\circ} 36^{\prime} 57^{\prime \prime} 7$ & 519 & 76 & 21.60 & 1.02 & 0.49 & 3.9 & 739 & \pm 17 & NGC 3384 cluster \\
\hline gc358 & $10^{\mathrm{h}} 48^{\mathrm{m}} 22^{\mathrm{s}} .79$ & $12^{\circ} 38^{\prime} 44^{\prime \prime} 2$ & 546 & 65 & 21.35 & 0.59 & 0.46 & 5.6 & 577 & \pm 13 & NGC 3384 cluster \\
\hline ad1021 & $10^{\mathrm{h}} 48^{\mathrm{m}} 13^{\mathrm{s}} .33$ & $12^{\circ} 42^{\prime} 24^{\prime \prime} 8$ & 573 & 38 & 21.71 & 0.99 & 0.52 & 5.3 & 752 & \pm 7 & NGC 3384 cluster? \\
\hline gc756 & $10^{\mathrm{h}} 47^{\mathrm{m}} 51^{\mathrm{s}} .58$ & $12^{\circ} 45^{\prime} 40^{\prime} \cdot 2$ & 646 & 2 & 20.87 & 0.58 & 0.47 & 3.7 & 725 & \pm 19 & Class B \\
\hline gc368 & $10^{\mathrm{h}} 48^{\mathrm{m}} 22^{\mathrm{s}} .19$ & $12^{\circ} 44^{\prime} 00^{\prime} .3$ & 731 & 42 & 21.15 & 0.82 & 0.44 & 4.6 & 1098 & \pm 10 & \\
\hline ad1481 & $10^{\mathrm{h}} 47^{\mathrm{m}} 52^{\mathrm{s}} .08$ & $12^{\circ} 21^{\prime} 45^{\prime \prime} .2$ & 790 & 177 & 19.39 & 0.52 & 0.43 & 7.0 & 1105 & \pm 6 & \\
\hline gc185 & $10^{\mathrm{h}} 48^{\mathrm{m}} 38^{\mathrm{s}} .75$ & $12^{\circ} 27^{\prime} 09^{\prime} 3$ & 870 & 122 & 21.09 & 0.64 & 0.37 & 4.8 & 964 & \pm 10 & \\
\hline
\end{tabular}

${ }^{a} \equiv$ Brodie \& Larsen (2002) N3384-FF-7 “fuzzy" cluster. They found $v=905 \pm 23 \mathrm{~km} \mathrm{~s}^{-1}$ (red channel value) using Keck/LRIS.

by comparison to the strong signal seen around NGC 3384, the overall rotation in the NGC 3379 GC system appears to be less than $100 \mathrm{~km} \mathrm{~s}^{-1}$ about any axis. This low rotation is consistent with that seen in the stars at small radii (e.g.
Statler \& Smecker-Hane 1999) and is similar to that seen in the GC system of the elliptical galaxy NGC 4472 (see Zepf et al. 2000; Côté et al. 2003) although probably less than that in NGC 5128 (Peng et al. 2004), a peculiar merger remnant. 


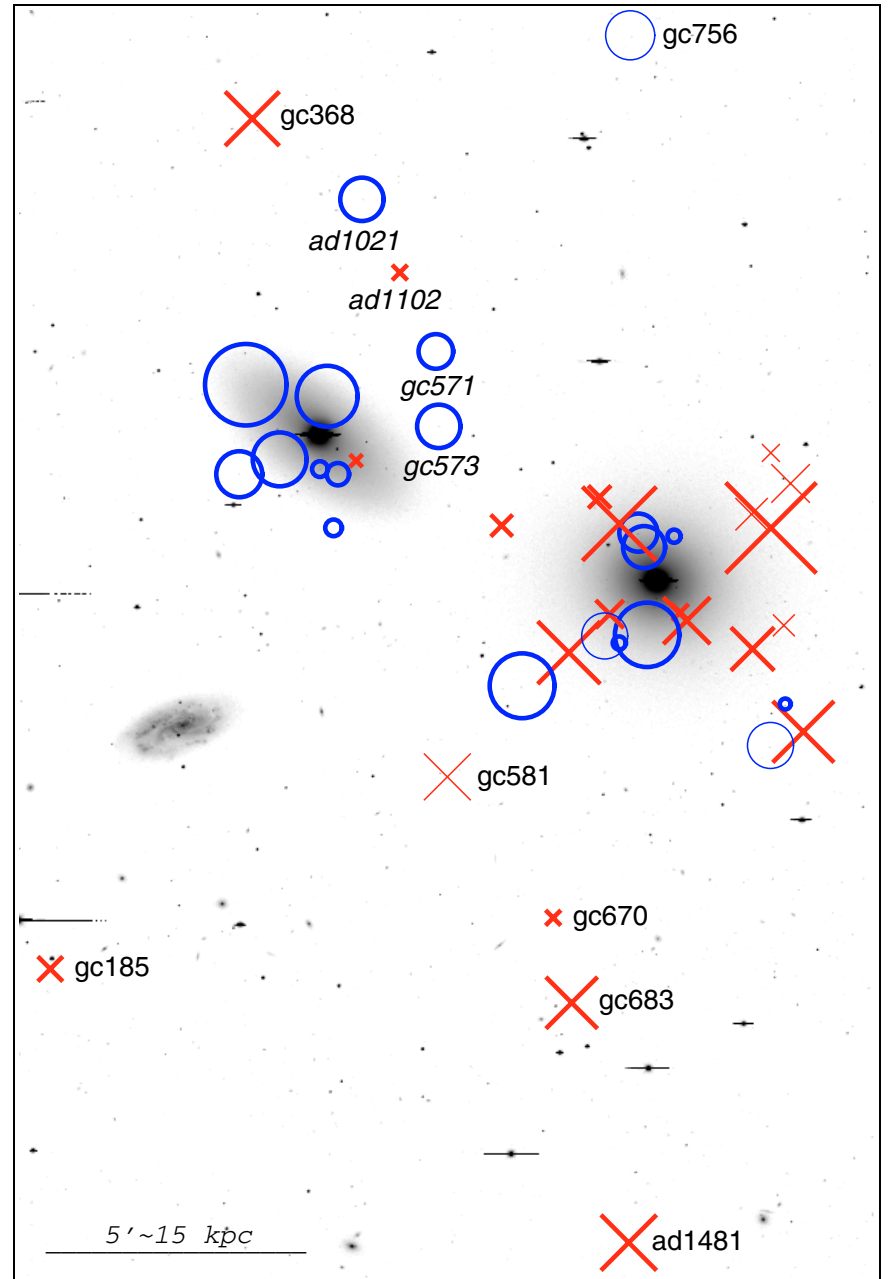

Fig. 3. Velocities of the 42 confirmed GCs overplotted on a $V$-band MOSAIC sub-image of $25^{\prime} \times 15^{\prime}$ (north is up, east to the left). Crosses $(\times)$ are GCs with $v>904 \mathrm{~km} \mathrm{~s}^{-1}$ which we adopt as the systemic velocity of NGC 3379. Circles ( $\bigcirc$ ) are those GCs with $v<904 \mathrm{~km} \mathrm{~s}^{-1}$; the symbol size is proportional to the offset, up to about $\pm 400 \mathrm{~km} \mathrm{~s}^{-1}$, with class A objects in boldface. Note the clear rotation of the eight GCs along the NGC 3384 disc and their lower typical velocities compatible with the systemic value of this SB0. The seven outer $\left(R>5^{\prime}\right)$ GCs associated with NGC 3379 and the four globulars with uncertain assignments to one of the two galaxies are identified individually, with the unassigned objects in italics.

\subsection{Kinematics of the globular cluster system of NGC 3384}

Our sample also has eight GCs that are clearly associated with NGC 3384. Even with just these eight radial velocities, there is evidence for rotation $(v / \sigma \sim 2)$ in the GC system of NGC 3384, which can be seen visually in Fig. 3. Specifically, the four GCs to the south-west of the galaxy centre have radial velocities $200 \mathrm{~km} \mathrm{~s}^{-1}$ lower than the four to the north-east. This rotation signature is very similar to that found by a study of $68 \mathrm{PNe}$ around this galaxy by Tremblay et al. (1995). The rotation in the GC system of NGC 3384 suggests that rotation may not be unusual in the GC systems of disc galaxies. This brings into question whether the lack of rotation in the large majority of the

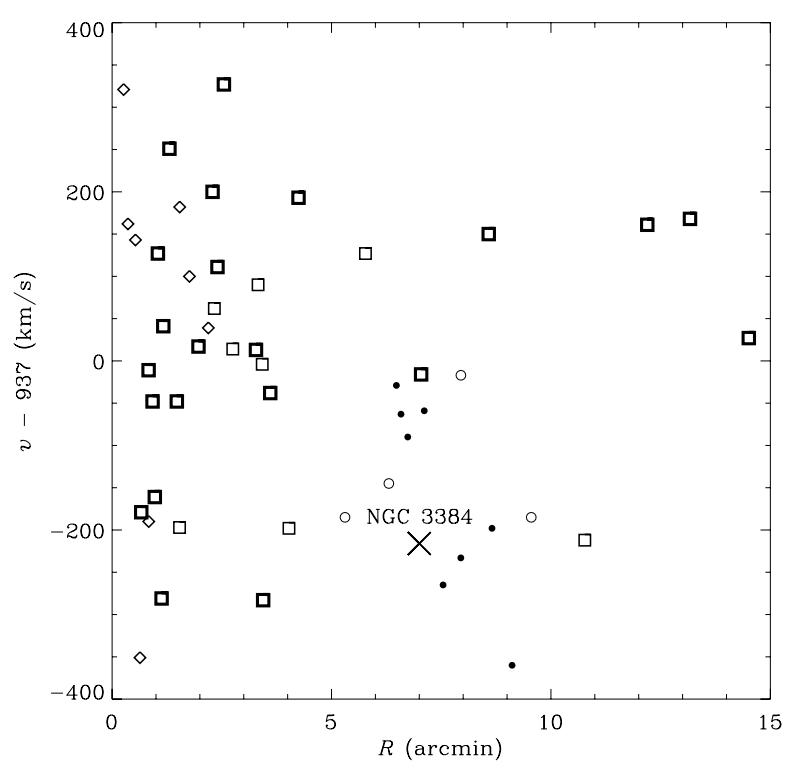

Fig. 4. Relative velocities vs. distances to NGC 3379 for the spectroscopic sample of 42 globular clusters. GCs associated with NGC 3379 are shown as squares (bold for class A; diamonds are eight additional GCs from P04), those associated with NGC 3384 as filled circles and GCs possibly associated with either galaxy as open circles.

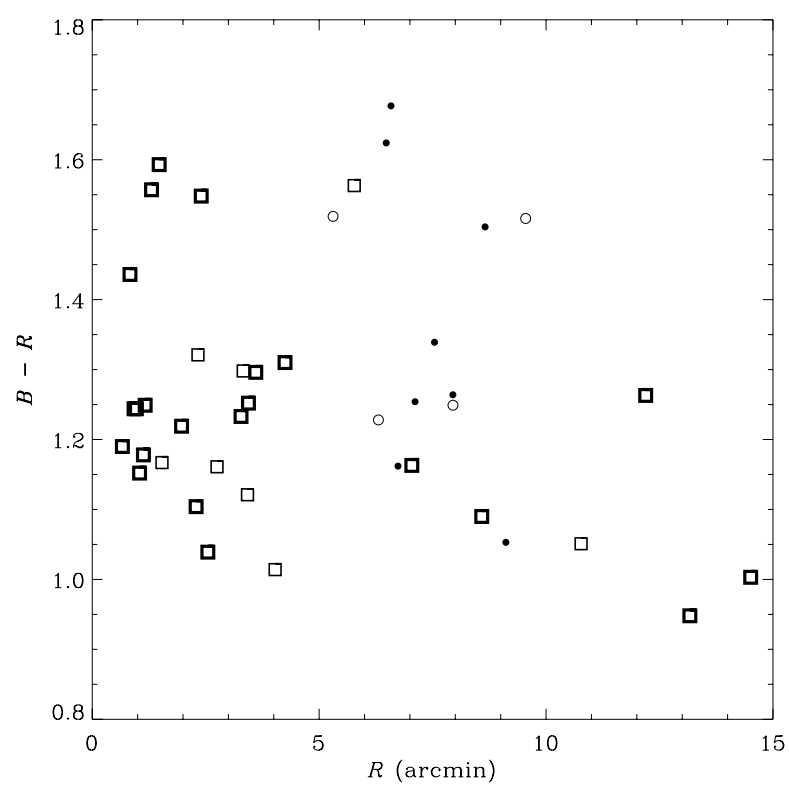

Fig. 5. $B-R$ colours vs. radii for our spectroscopic sample of $42 \mathrm{GCs}$ (symbols are as in Fig. 4). The plot shows the trend of redder colours for GCs close to the NGC 3379 centre and bluer colours at large radii.

Milky Way globular cluster system (e.g. Zinn 1985) is somewhat unusual, and the rotation seen in the Messier 31 GC system (e.g., Perrett et al. 2002) is possibly more typical.

\subsection{Colours of the globular clusters around NGC 3379 and NGC 3384}

We can use our dataset to examine questions about the colours of the NGC 3379 and NGC 3384 GCs with a spectroscopically 
confirmed dataset. In Fig. 5 we plot the $B-R$ colours from RZ04 of our GC sample as a function of their distance from the centre of NGC 3379. The data show that NGC 3379 has more blue, metal-poor GCs than red metal-rich ones, in agreement with the suggestion of RZ04 from their photometry. As noted in that paper, of current models for the formation of GC systems, only those that involve galaxy mergers (e.g. Ashman \& Zepf 1992; Beasley et al. 2002) are readily able to account for this larger number of blue relative to red GCs in an elliptical galaxy like NGC 3379 with its modest luminosity and small GC population.

The data shown in Fig. 5 also indicate a colour gradient in the GC system of NGC 3379, such that the ratio of blue to red GCs increases with distance away from the galaxy centre. This is consistent with RZ04 and many other previous studies for the globular cluster systems of a number of elliptical galaxies (see Ashman \& Zepf 1998, for a review). However, RZ04 also noted that the photometry indicated that there may be red, metal-rich GCs at large distances around some elliptical galaxies. In NGC 3379, our data show a metal-rich GC (gc581) at a projected distance of almost $6^{\prime}$, corresponding to about $17 \mathrm{kpc}$. The GC ad1021 is also interesting in this regard, as it is very red, and is almost $10^{\prime}$ away from NGC 3379 and about $5^{\prime}$ away from NGC 3384. Thus there are two spectroscopically confirmed, metal-rich clusters well into the halos of these galaxies. We also note that two of the inner GCs associated with NGC 3384 are very red, which might be attributable to dust in the system.

\section{Constraints on the halo of NGC 3379}

\subsection{Velocity dispersion profile}

One of the primary goals of this study is to determine the radial velocities of GCs to probe the halo dynamics of NGC 3379. To test mass models of the halo of NGC 3379, we use our dataset to determine the projected velocity dispersion at several different radii. As shown in Fig. 6, we adopt three radial bins to allow the comparison of the mass over a range of radii, corresponding in the inner region to the largest radial extent of the stellar data, in the intermediate region to the largest extent of the $\mathrm{PNe}$ data, and the outer region which is uniquely probed by our globular cluster dataset. We then plot the projected velocity dispersions $\sigma_{\mathrm{p}}$ in these three radial regions in Fig. 6. To calculate $\sigma_{\mathrm{p}}$, the mean velocity of $v_{0}=937 \mathrm{~km} \mathrm{~s}^{-1}$ is adopted, and the measurement uncertainties $\delta v_{i}$ are taken into account. The $\pm 34 \%$ uncertainties are estimated by 30000 Monte Carlo simulated data sets drawn from the best-fit model. Note that the dispersion $\sigma_{\mathrm{p}}$ discussed throughout this paper incorporates any rotational support, and thus is technically $v_{\mathrm{rms}}=\left(v_{\mathrm{p}}^{2}+\sigma_{\mathrm{p}}^{2}\right)^{1 / 2}$.

Figure 6 shows the binned velocity dispersion profile. We estimate $\sigma_{\mathrm{p}}=205_{-40}^{+43} \mathrm{~km} \mathrm{~s}^{-1}, 155_{-26}^{+27} \mathrm{~km} \mathrm{~s}^{-1}$ and $147_{-39}^{+44} \mathrm{~km} \mathrm{~s}^{-1}$ at $R \sim 0$ '. $^{\prime}(13 \mathrm{GCs}), \sim 2$ '.5 (18 GCs) and $\sim 11^{\prime}$ (7 GCs), respectively. Inclusion of the four uncertain GCs has very little effect: the outer $\sigma_{\mathrm{p}}$ becomes $147_{-32}^{+33} \mathrm{~km} \mathrm{~s}^{-1}$. Alternatively adopting $904 \mathrm{~km} \mathrm{~s}^{-1}$ for the NGC 3379 velocity has the effect of raising the outer two $\sigma_{\mathrm{p}}$ estimates to $165_{-27}^{+29} \mathrm{~km} \mathrm{~s}^{-1}$ and $165_{-44}^{+48} \mathrm{~km} \mathrm{~s}^{-1}$.

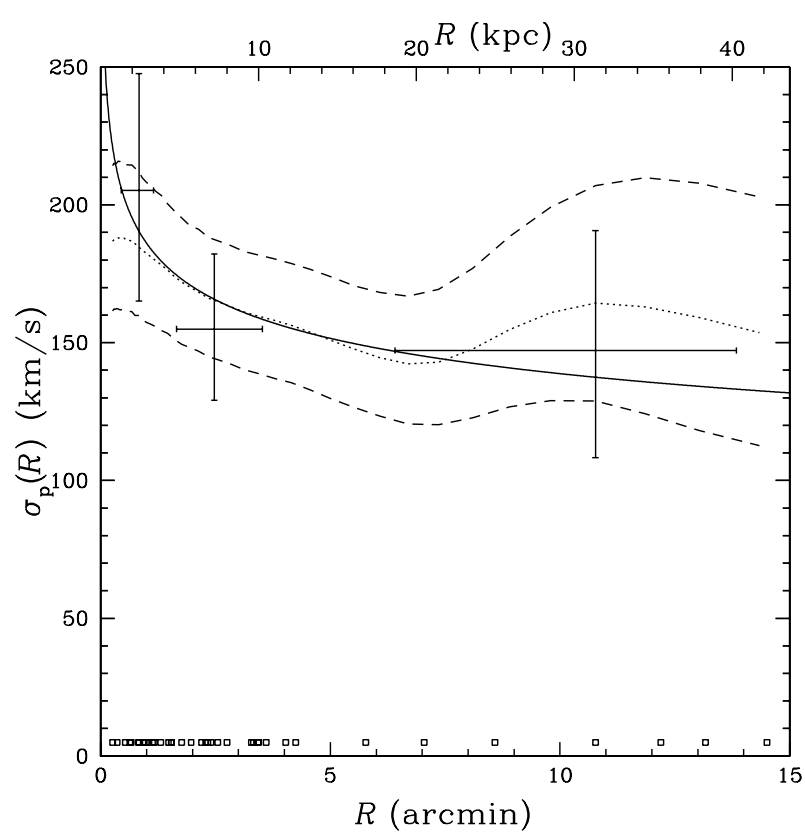

Fig. 6. Projected velocity dispersion vs. radius for the NGC 3379 GCs. The error bars show radially-binned data, where the vertical bars are for the $\pm 34 \%$ uncertainties, and the horizontal bars show the range covered by $68 \%$ of the data. The dotted line shows a moving window estimate, with dashed lines signifying the $\pm 34 \%$ uncertainties. The solid line shows the best-fit scale-free power-law profile. The boxes at the bottom indicate the radial positions of the GC velocity data.

We also construct a smoothed estimate of $\sigma_{\mathrm{p}}(R)$, using a "moving window". This performs a $\sigma_{\mathrm{p}}$ estimate at every radius $R$ using the nearby data (each estimate is thus not independent). Unlike previous versions of this method which used a window of constant width (Zepf et al. 2000; Côté et al. 2001) or with fixed number of data points (Kissler-Patig \& Gebhardt 1998; Cohen 2000), we use an exponential weighting function for the contribution of each data point $v_{i}$ to the estimate at radius $R$ :

$w_{i}(R)=\frac{1}{\sigma_{R}} \exp \left[\frac{\left(R-R_{i}\right)^{2}}{2 \sigma_{R}^{2}}\right]$,

where the half-width $\sigma_{R} \propto[R \Sigma(R)]^{-1}$, and $\Sigma(R)$ is the underlying surface density distribution from which the measurements are drawn. For "perfect" sampling, the number of points contributing to each estimate is constant. For optimal smoothing, the number of points within $\pm \sigma_{R}$ has a maximum of $\sim 25$. For the current data set, the surface density is the $R^{1 / 4}$ law found by RZ04, and the window width is normalised to $\sigma_{R} \simeq 1$ '.8.

Figure 6 shows the result of this procedure. The dispersion profile is consistent with being constant over the wide range of radii studied, with a possible increase to small radii. The inclusion of the four uncertain GCs does not affect the profile much, but a mean velocity of $904 \mathrm{~km} \mathrm{~s}^{-1}$ would raise $\sigma_{\mathrm{p}}$ in most places by $10-20 \mathrm{~km} \mathrm{~s}^{-1}$. Utilising a maximum likelihood technique for fitting the data to a scale-free power-law (see Appendix A), we also find the same result that the velocity dispersion is generally constant with radius with a possible modest increase in the dispersion to small radii. 


\subsection{Halo models}

A primary goal of this programme is to test whether our measured velocity dispersion profile for the GC system of NGC 3379 is consistent with the mass distribution expected from standard $\Lambda \mathrm{CDM}$ halos or other models. To perform this test, we construct mass models of NGC 3379 with and without dark matter halos. The circular velocity of these models is:

$v_{\mathrm{c}}^{2}(r)=\frac{v_{\star}^{2} a_{\star} r}{\left(r+a_{\star}\right)^{2}}+v_{\mathrm{s}}^{2}\left[\frac{r_{\mathrm{s}}}{r} \ln \left(1+\frac{r}{r_{\mathrm{s}}}\right)-\frac{r_{\mathrm{s}}}{r+r_{\mathrm{s}}}\right]$,

where $v_{\star}$ and $a_{\star}$ describe the stellar mass distribution (after Hernquist 1990) and $v_{\mathrm{s}}$ and $r_{\mathrm{s}}$ are the Navarro et al. (1996) NFW parameters of the dark matter halo. More accurate representations of the stellar and dark matter distributions are possible (e.g. Mamon \& Łokas 2005), but at this stage we wish to use identical models to R03 for consistent comparisons.

For the stellar mass distribution, extensive studies of stellar dynamics in the inner regions of NGC 3379 (Kronawitter et al. 2000; Gebhardt et al. 2000; Cappellari et al. 2005), find stellar mass-to-light ratios of $\Upsilon_{\star, B}=5$-6 (in units of $\Upsilon_{\odot, B}$ ). These models neglect any dark matter found inside $R_{\mathrm{e}}$, so the actual $\Upsilon_{\star, B}$ may be lower. The orbit models of R03 separate $M(r)$ into the stellar and dark components, giving $\Upsilon_{\star, B}=6$ (based primarily on the kinematics of the stars rather than the $\mathrm{PNe}$ ). Stellar population synthesis models with a Kroupa IMF imply $\Upsilon_{\star, B}=4-7$ (Gerhard et al. 2001; Napolitano et al. 2005; Cappellari et al. 2005). We adopt $\Upsilon_{\star, B}=5.8$ as the overall best estimate, and combine this with the observed light profile to construct the galaxy mass profile. The resulting stellar mass models for NGC 3379 gives $v_{\star}=603 \mathrm{~km} \mathrm{~s}^{-1}$ and $a_{\star}=0.3$ $\left(R_{\mathrm{e}}=0.6\right)$ for use in Eq. (2) above.

To determine the $v_{s}$ and $r_{s}$ values for a given $\Lambda \mathrm{CDM}$ halo, we compute the virial mass and radius, $M_{\mathrm{vir}}$ and $r_{\mathrm{vir}}$, and express the model halos in terms of $M_{\mathrm{vir}}$ and the concentration $c_{\text {vir }} \equiv r_{\text {vir }} / r_{\mathrm{s}}$. In $\Lambda \mathrm{CDM}$, there is a welldetermined mean relation between these parameters: $c_{\mathrm{vir}} \simeq$ $17.9\left(M_{\text {vir }} / 10^{11} M_{\odot}\right)^{-0.125}$, with a $68 \%$ log scatter of 0.14 (Bullock et al. 2001; Napolitano et al. 2005). Our approach here differs from R03 in assuming a fluctuation amplitude $\sigma_{8}=0.9$ rather than 1.0, and in using a virial overdensity of 101 rather than 200 - changes that permit less massive inner halos in $\Lambda \mathrm{CDM}$.

We then consider whether $\Lambda \mathrm{CDM}$ halos with various masses and concentrations are consistent with the velocity dispersions we find for the NGC 3379 GCs. To do this, we calculate the projected velocity dispersion of the globular cluster system using a Jeans equation:

$\sigma_{\mathrm{p}}^{2}(R)=\frac{2}{\Sigma(R)} \int_{R}^{\infty} \frac{r \mathrm{~d} r}{\sqrt{r^{2}-R^{2}}} \int_{r}^{\infty} \frac{j\left(r^{\prime}\right) v_{\mathrm{c}}^{2}\left(r^{\prime}\right) \mathrm{d} r^{\prime}}{r^{\prime}}$.

In making this calculation of the $\sigma_{\mathrm{p}}(R)$ expected for the GC system given a mass model for the galaxy, we assume the following: 1) spherical symmetry; 2) isotropic distribution function and Gaussian LOSVDs; 3) density distribution of tracers $j(r)$ well-known; 4) dynamically negligible gas content; 5) stellar mass-to-light ratio $\Upsilon_{\star}$ well-constrained by other techniques.

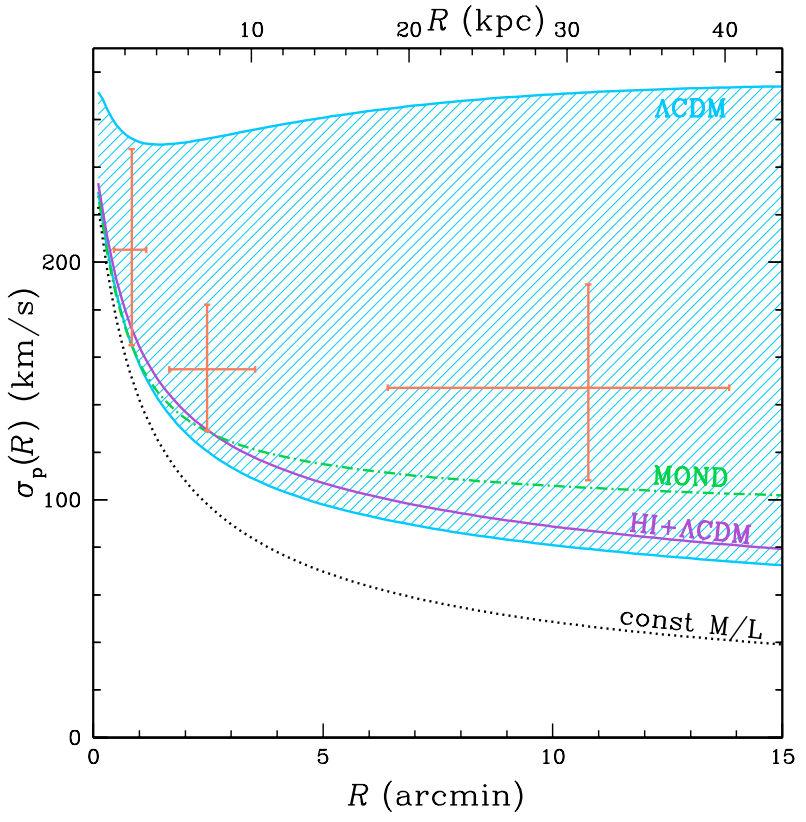

Fig. 7. Model predictions compared to the observed velocity dispersion profile of the NGC 3379 GCs. Error bars are as described in Fig. 6. The hatched region describes profiles for typical $\Lambda$ CDM halos as described in the text. The solid line shows the "consensus" model that matches the HI data and provides a reasonable fit to the GC and PNe results. The dotted line represents a mass traces light model with Newtonian dynamics, while the dot-dashed line is with MOND.

The spherical assumption is motivated by the roundness of NGC 3379 (e.g. Peletier et al. 1990), the absence of evidence for substantial flattening of the GC system of NGC 3379 (RZ04), and the evidence that globular cluster systems appear to be typically at least as round as their host galaxies (e.g., Ashman \& Zepf 1998). Similarly, isotropy is observed for systems where sufficient data are available (e.g. Zepf et al. 2000; Côté et al. 2003); our $\hat{\sigma}_{\mathrm{p}}$ fits to the NGC 3379 GC velocities give very similar results to traditional $\sigma_{\mathrm{p}}$ calculations, indicating that Gaussianity is a good approximation. The density distribution we adopt is a Hernquist (1990) model which matches the $R^{1 / 4}$ law fitted to the NGC 3379 globular clusters (RZ04): $j(r) \propto r^{-1}(r+a)^{-3}$, where $a=9{ }^{\prime} 8\left(R_{\mathrm{e}}=17 ! 9\right)$. The mass of hot gas within $100 \mathrm{kpc}$ of NGC 3379 is no more than $\approx 10^{8} M_{\odot}$ (David et al. 2005, conservatively assuming a linear increase in gas mass from the inner parts), which is a negligible fraction of the stellar mass of $M_{\star} \approx 10^{11} M_{\odot}$.

\subsection{Comparison of globular cluster system velocity dispersion to models}

The comparison of the $\sigma_{\mathrm{p}}(R)$ of the GC data to predictions for various models of the mass distribution for NGC 3379 is shown in Fig. 7. The GC data are in good agreement with a wide variety of $\Lambda C D M$ halos that have typical concentrations for their masses and match the inner stellar data. In detail, the range of $\Lambda \mathrm{CDM}$ halos plotted is based on halos which fall within the $1 \sigma$ scatter in the halo mass-concentration relation of Bullock et al. (2001), with the additional constraints that the stellar mass to 
dark halo mass is not lower than typical for galaxy clusters and is not larger than the cosmic baryon fraction.

A valuable constraint on the halo mass of NGC 3379 is provided by observations of a rotating ring of HI gas at $R$ $100 \mathrm{kpc}$ (Schneider et al. 1983; Schneider 1985). The positions and velocities of the $\mathrm{HI}$ are consistent with the gas being in a Keplerian orbit around the barycentre of NGC 3379 and NGC 3384 , with about $2 / 3$ of the mass within $100 \mathrm{kpc}$ belonging to NGC 3379 . This then gives a mass for NGC 3379 of about $4-5 \times 10^{11} M_{\odot}$ at $R \sim 100 \mathrm{kpc}$ and $\Upsilon_{B} \simeq 25-35$. A $\Lambda \mathrm{CDM}$ halo model that matches the $\mathrm{HI}$ ring and is generally consistent with the GC and PN dynamical constraints is shown in Fig. 7. This "consensus" model predicts a GC velocity dispersion that is close to that observed, with a preference for smaller values.

This "best-fitting" $\Lambda$ CDM halo is only slightly below the GC data and has a concentration well within the normal range for a halo of its mass. The only notable feature of this $\Lambda \mathrm{CDM}$ halo is that it gives a fairly small mass-to-light ratio, with $\Upsilon_{B} \simeq 35$. Modest mass-to-light ratios for early-type galaxies with luminosities around $L^{*}$ like NGC 3379 may be in agreement with expectations from some recent halo occupation models (e.g. van den Bosch et al. 2003), although the value for NGC 3379 is about a factor of two less than the model average for galaxies of its mass. Another implication of the inferred mass-to-light ratio is that if the halo has the universal baryonic fraction of 0.17 of the concordance model, and given the $\Upsilon_{B}$ of about 6 measured in the inner regions, the $\Upsilon_{B}$ for the halo would suggest most of the baryons have been turned into stars (see Napolitano et al. 2005, for this calculation for a number of galaxies).

Our data also allow us to assess alternatives to $\Lambda \mathrm{CDM}$ halos. Assuming no dark matter and a Newtonian force law, we expect $\sigma_{\mathrm{p}}=98 \mathrm{~km} \mathrm{~s}^{-1}$ at $R=2.5$ and $49 \mathrm{~km} \mathrm{~s}^{-1}$ at $10^{\prime}$. This mass traces light model is clearly ruled out by the data (see Fig. 7). We also consider MOND, which has been proposed to provide a good fit to the earlier PNe data (Milgrom \& Sanders 2003). The comparison of our observed $\sigma_{\mathrm{p}}$ with the predictions of MOND is shown in Fig. 7. The velocity dispersion of the GCs is slightly higher than the MOND prediction, although the uncertainties mitigate against an unambiguous rejection of MOND.

A final comparison is to the published velocity dispersions of NGC 3379 PNe (R03). The reasonable agreement of all of the constraints with the overall "best-fit" mass model indicates that the GCs and PNe are broadly consistent. In detail, although the PNe have a $\sigma_{\mathrm{p}}$ of about $120 \mathrm{~km} \mathrm{~s}^{-1}$ in the radial range of $0.7 \leq R \leq 4^{\prime}$ where the PN data overlap with our GC data, compared to a $\sigma_{\mathrm{p}}$ of about $170 \mathrm{~km} \mathrm{~s}^{-1}$ for the GCs in the same region, the two do not give greatly discrepant masses. This is partly due to radial anisotropy in the PNe system suggested by orbital modeling of the PN system (R03), which naturally gives a somewhat lower $\sigma_{\mathrm{p}}$ for the same mass, and partly due to the uncertainties in $\sigma_{\mathrm{p}}$ from the modest number of radial velocities. Furthermore, the GCs appear to have a shallower spatial profile than the galaxy light (and by inference the PNe) in the relevant region (RZ04) which would produce a smaller $\sigma_{\mathrm{p}}$ for the $\mathrm{PNe}$ relative to the GCs. If additional data find a difference in the $\sigma_{\mathrm{p}}$ beyond these effects, several possibilities present themselves. These include the possibility that the GCs may have modestly tangential orbits, that orbits may be disturbed by group interactions, or that NGC 3379 may have a substantial disc component seen face-on (e.g. Capaccioli et al. 1991) which would be more likely to include PNe than GCs. The question of the halo mass profile inferred for NGC 3379 has also been addressed in a recent paper by Dekel et al. (2005). They simulated disk galaxy mergers and found that in the resulting elliptical galaxies, tracers like PNe have steeply declining $\sigma_{\mathrm{p}}$ profiles because of radially biased orbits, flattened triaxial structures, and steep spatial profiles. They predicted that GCs would be less prone to these effects and should have flatter dispersion profiles, which is consistent with our results.

\section{Conclusions}

We have used the FLAMES multi-fibre spectrograph on the VLT to obtain radial velocities of globular clusters (GCs) around the Leo I group galaxies NGC 3379 and NGC 3384. FLAMES allowed us to obtain spectra of 42 GCs over a wide field $\left(1^{\prime} \lesssim r \lessgtr 14^{\prime}\right)$ with very small uncertainties $\left(\sim 10 \mathrm{~km} \mathrm{~s}^{-1}\right)$.

1. Our primary conclusion is that the projected velocity dispersion of the GC system of NGC 3379 is consistent with that expected from standard dark matter halos in $\Lambda \mathrm{CDM}$ cosmologies. This is based on comparing our observed velocity dispersions for the GC system over a wide range of radii to the expectation from the Jeans equation for the mass profile of $\Lambda \mathrm{CDM}$ halo models and the observed radial profile of the GC system.

2. We find evidence that the GC system of the SB0 galaxy NGC 3384 has a rotation similar to that of the stellar disc and planetary nebula system of this galaxy. This result suggests that significant rotation in the globular cluster systems of disc galaxies may not be rare.

3. We find a colour gradient in our spectroscopic sample such that the fraction of red, metal-rich GCs decreases from small to large radii, in agreement with photometric studies. Even with this colour gradient, we also find some red GCs at large distances from the centre of their host galaxy, which may place interesting constraints on models for the origin of metal-rich globular clusters.

Acknowledgements. G.B. is supported by the Secretaría de Estado de Universidades e Investigación with additional support by DGI (Spain) AYA 2002-03338 and Junta de Andalucía TIC-114. S.E.Z. acknowledges support for this work in part from NSF AST-0406891 and from the Michigan State University Foundation. A.J.R. is supported by the FONDAP Center for Astrophysics Conicyt 15010003. K.L.R. is supported by an NSF Astronomy and Astrophysics Postdoctoral Fellowship under award AST-0302095.

\section{Appendix A: Fitting dispersion profiles to discrete velocity data}

Radial binning or smoothing is not ideal for characterising data and testing models, as the grouping of data discards useful information about the radial locations $R_{i}$ and may skew the result 
by assuming $\sigma_{\mathrm{p}}$ is constant within each bin. As an alternative, we consider a maximum-likelihood technique for fitting discrete velocity data to local velocity dispersions (see Ciardullo et al. 1993; Saglia et al. 2000). This technique allows one to fit data to a model for $\sigma_{\mathrm{p}}(R)$, which may be e.g. a constant dispersion, a power-law, or the prediction of a Jeans model.

We convolve a Gaussian line-of-sight velocity distribution (LOSVD) with a Gaussian measurement function $v_{i} \pm \delta v_{i}$ to derive the likelihood of a given measurement $v_{i}$ given a model dispersion $\sigma_{\mathrm{p}}\left(R_{i}\right)$ :

$$
\mathcal{L}_{i}=\frac{1}{\sqrt{\sigma_{\mathrm{p}}^{2}+\left(\delta v_{i}\right)^{2}}} \exp \left\{\frac{\left(v_{i}-v_{0}\right)^{2}}{2\left[\sigma_{\mathrm{p}}^{2}+\left(\delta v_{i}\right)^{2}\right]}\right\}
$$

which can be converted into a $\chi^{2}$ statistic:

$\chi^{2}=\sum_{i=1}^{i=N}\left\{\frac{\left(v_{i}-v_{0}\right)^{2}}{\sigma_{\mathrm{p}}^{2}+\left(\delta v_{i}\right)^{2}}+\ln \left[\sigma_{\mathrm{p}}^{2}+\left(\delta v_{i}\right)^{2}\right]\right\}$.

This method assumes Gaussian LOSVDs, so strictly speaking, we are computing the best-fit Gaussian parameter $\hat{\sigma}_{\mathrm{p}}$ rather than the true second-moment $\sigma_{\mathrm{p}}$. For near-isotropic systems, $\hat{\sigma}_{\mathrm{p}} \simeq \sigma_{\mathrm{p}}$.

To apply this to our globular cluster data for NGC 3379, we characterise the dispersion data by a scale-free power-law profile:

$\sigma_{\mathrm{p}}^{2}(R)=\sigma_{0}^{2}\left(R / R_{0}\right)^{-\gamma_{\mathrm{p}}}$,

where $R_{0}=2 ! 2$. We find $\sigma_{0}=168_{-19}^{+21} \mathrm{~km} \mathrm{~s}^{-1}$ and $\gamma_{\mathrm{p}}=0.25 \pm$ 0.25 (see Fig. 6). This result is scarcely affected by including the four uncertain clusters; on the other hand, adopting a mean velocity of $904 \mathrm{~km} \mathrm{~s}^{-1}$ would mean $\sigma_{0}=177_{-21}^{+21} \mathrm{~km} \mathrm{~s}^{-1}$ and $\gamma_{\mathrm{p}}=0.20 \pm 0.25$.

This dispersion estimator is checked using Monte Carlo simulations. A small correction for estimator bias is derived using the difference between the simulations' input and median output. The (doubly-debiassed) 68\% scatter of the simulations gives the likely range of the dispersion.

\section{References}

Ashman, K. M., \& Zepf, S. E. 1992, ApJ, 384, 50

Ashman, K. M., \& Zepf, S. E. 1998, Globular Cluster Systems (Cambridge University Press)

Beasley, M. A., Baugh, C. M., Forbes, D. A., Sharples, R. M., \& Frenk, C. S. 2002, MNRAS, 333, 383

Brainerd, T. G. 2005, ApJ, submitted [arXiv: astro-ph/0409381]

Brodie, J. P., \& Larsen, S. S. 2002, AJ, 124, 1410

Bullock, J. S., Kolatt, T. S., Sigad, Y., et al. 2001, MNRAS, 321, 559

Capaccioli, M., Vietri, M., Held, E. V., \& Lorenz, H. 1991, ApJ, 371, 535

Cappellari, M., Bacon, R., Bureau, M., et al. 2005, MNRAS, submitted [arXiv: astro-ph/0505042]

Ciardullo, R., Jacoby, G. H., \& Dejonghe, H. B. 1993, ApJ, 414, 454

Cohen, J. G. 2000, AJ, 119, 162

Côté, P., McLaughlin, D. E., Hanes, D. A., et al. 2001, ApJ, 559, 828

Côté, P., McLaughlin, D. E., Cohen, J. G., \& Blakeslee, J. P. 2003, ApJ, 591, 850
David, L. P., Jones, C., Forman, W., \& Murray, S. 2005, ApJ, 635, 1053

Dekel, A., Stoehr, F., Mamon, G. A., et al. 2005, Nature, 437, 707

Falco, E. E., Kurtz, M. J., Geller, M. J., et al. 1999, PASP, 111, 438

Gebhardt, K., Richstone, D., Kormendy, J., et al. 2000, AJ, 119, 1157

Gerhard, O., Kronawitter, A., Saglia, R. P., \& Bender, R. 2001, AJ, 121,1936

Gregg, M. D., Ferguson, H. C., Minniti, D., Tanvir, N., \& Catchpole, R. 2004, AJ, 127, 1441

Harris, G. L. H., Harris, W. E., \& Geisler, D. 2004, AJ, 128, 723

Hernquist, L. 1990, ApJ, 356, 359

Hoekstra, H. M., Yee, H. K. C., \& Gladders, M. D. 2004, ApJ, 606, 67

Jensen, J. B., Tonry, J. L., Barris, B. J., et al. 2003, ApJ, 583, 712

Kissler-Patig, M., \& Gebhardt, K. 1998, AJ, 116, 2237

Kronawitter, A., Saglia, R. P., Gerhard, O., \& Bender, R. 2000, A\&AS, 144,53

Loewenstein, M., \& White, R. E. 1999, ApJ, 518, 50

Mamon, G. A., \& Łokas, E. W. 2005, MNRAS, 363, 705

McKay, T. A., Sheldon, E. S., Racusin, J., et al. 2001 [arXiv: astro-ph/0108013]

Milgrom, M., \& Sanders, R. H. 2003, ApJ, 599, L25

Minniti, D., \& Rejkuba, M. 2002, ApJ, 575, L59

Moultaka, J., Ilovaisky, S., Prugniel, Ph., \& Soubiran, C. 2004, PASP, 116,693

Napolitano, N. R., Capaccioli, M., Romanowsky, A. J., et al. 2005, MNRAS, 357, 691

Navarro, J. F., Frenk, C. S., \& White, S. D. M. 1996, ApJ, 462, 563 (NFW)

Pasquini, L., Castillo, R., Dekker, H., et al. 2004, SPIE, 5492, 136

Peletier, R. F., Davies, R. L., Illingworth, G. D., Davis, L. E., \& Cawson, M. 1990, AJ, 100, 1091

Peng, E. W., Ford, H. C., \& Freeman, K. C. 2004, ApJ, 602, 705

Perrett, K. M., Bridges, T. J., Hanes, D. A., et al. 2002, AJ, 123, 2490

Prada, F., Vitvitska, M., Klypin, A., et al. 2003, ApJ, 598, 260

Puzia, T. H., Kissler-Patig, M., Thomas, D., et al. 2004, A\&A, 415, 123 (P04)

Romanowsky, A. J., \& Kochanek, C. S. 2001, ApJ, 553, 722

Rhode, K. L., \& Zepf, S. E. 2001, AJ, 121, 210

Rhode, K. L., \& Zepf, S. E. 2004, AJ, 127, 302 (RZ04)

Richtler, T., Dirsch, B., Gebhardt, K., et al. 2004, AJ, 127, 2094

Romanowsky, A. J., Douglas, N. G., Arnaboldi, M., et al. 2003, Science, 301, 1696 (R03)

Saglia, R. P., Kronawitter, A., Gerhard, O., \& Bender, R. 2000, AJ, 119,153

Schneider, S. E., Helou, G., Salpeter, E. E., \& Terzian, Y. 1983, ApJ, 273, L1

Schneider, S. E. 1985, ApJ, 288, L33

Smith, R. J., Lucey, J. R., Hudson, M. J., Schlegel, D. J., \& Davies, R. 2000, MNRAS, 313, 469

Statler, T. S. 1994, AJ, 108, 111

Statler, T. S., \& Smecker-Hane, T. 1999, AJ, 117, 839

Statler, T. S. 2001, AJ, 121, 244

Tonry, J., \& Davis, M. 1979, AJ, 84, 1511

Tremblay, B., Merritt, D., \& Williams, T. B. 1995, ApJ, 443, L5

Treu, T., \& Koopmans, L. V. E. 2004, ApJ, 611, 739

van den Bosch, F. C., Mo, H. J., \& Yang, X. 2003, MNRAS, 345, 923

Wilson, G., Kaiser, N., Luppino, G. A., \& Cowie, L. L. 2001, ApJ, 555,572

Zepf, S. E., Beasley, M. A., Bridges, T. J., et al. 2000, AJ, 120, 2928

Zinn, R. 1985, ApJ, 293, 424 